\title{
Superradiance Effects in the Linear and Nonlinear Optical Response of Quantum Dot Molecules
}

\author{
A. Sitek AND P. Machnikowski \\ Institute of Physics, Wrocław University of Technology \\ Wybrzeże Wyspiańskiego 27, 50-370 Wrocław, Poland
}

We calculate the linear optical response from a single quantum dot molecule and the nonlinear, four-wave-mixing response from an inhomogeneously broadened ensemble of such molecules. We show that both optical signals are affected by the coupling-dependent superradiance effect and by optical interference between the two polarizations. As a result, the linear and nonlinear responses are not identical.

PACS numbers: 78.67.Hc, 78.47.nj, 42.50.Ct

\section{Introduction}

Optical experiments are an efficient tool for the investigation of semiconductor nanostructures. In particular, the structure of energy levels and decoherence properties of quantum dots (QDs) can be determined from the linear and nonlinear optical response of these systems. While experimental methods based on the linear response are limited to single dots, nonlinear techniques, based on the photon echo effect, can be used to overcome the inhomogeneous broadening in QD ensembles and to extract useful information on the properties of individual dots.

In this paper, we study theoretically the linear and nonlinear four-wave mixing (FWM) optical response from a sample containing a non-homogeneously broadened ensemble of vertically stacked pairs of quantum dots (quantum dot molecules, QDMs) interacting with the quantized radiation field (vacuum). QDMs attract much interest since they show many interesting physical properties that are not present in single dots, like excitation transfer $[1,2]$ or superradiant luminescence $[3,4]$. We calculate the linear coherent polarization of a single QDM and the third-order polarization of a QDM ensemble, described by a statistical distribution of (possibly correlated) fundamental transition energies of the two dots in each QDM, as well as by a strength of the inter-dot coupling within a QDM (of dipole or tunneling origin). We show that signatures of superradiance can be observed in both kinds of experiments, even if the dots are not identical, provided that 
coupling between the dots is strong enough. Additional features, related to the dephasing of optical beats, appear at short time scales in the FWM response of coupled QDMs.

\section{The model}

The model under consideration is similar to that used in our previous work [5], except that coupling to the electromagnetic modes, leading to radiative decay, is now included. Each QDM is composed of two QDs with energies $E_{1,2}=E \pm \Delta$ and is modeled as a four-level system with the basis states $|00\rangle,|01\rangle$, $|10\rangle,|11\rangle$, corresponding to the ground state (empty dots), an exciton in the second and first QD, and excitons in both QDs, respectively. We describe its evolution in a frame rotating with the frequency $E / \hbar$. We write the Hamiltonian for the single QDM in the rotating wave approximation as $H=H_{\mathrm{X}}+H_{\mathrm{L}}+H_{\mathrm{rad}}+H_{\text {phot }}$.

The first component describes the excitons

$$
H_{\mathrm{X}}=\Delta(|1\rangle\langle 1|\otimes \mathbb{I}-\mathbb{I} \otimes| 1\rangle\langle 1|)+V(|01\rangle\langle 10|+| 10\rangle\langle 01|),
$$

where $|0\rangle,|1\rangle$ denote the states without and with an exciton in a dot, two-digit kets $|10\rangle,|01\rangle$ represent configurations with a single exciton in the first and second dot in the double-dot system, respectively, the tensor product refers to the two QDs making up the molecule, $V$ is the coupling between the dots, and $\mathbb{I}$ is the unit operator. Biexciton shift is neglected.

The second term in the Hamiltonian accounts for the interaction with the laser field, which is treated classically,

$$
H_{\mathrm{L}}=\frac{1}{2} \sum_{i} f_{i}(t)\left[\mathrm{e}^{-\mathrm{i}\left(\phi_{i}+E t_{i}\right)}(|0\rangle\langle 1|\otimes \mathbb{I}+\mathbb{I} \otimes| 0\rangle\langle 1|)+\text { h.c. }\right],
$$

where $f_{i}$ and $\phi_{i}$ are the amplitude envelopes and phases of the laser pulses, respectively.

The third term accounts for the interaction with the electromagnetic (EM) field in the dipole approximation,

$$
H_{\mathrm{rad}}=(|0\rangle\langle 1|\otimes \mathbb{I}+\mathbb{I} \otimes| 0\rangle\langle 1|) \sum_{\boldsymbol{k}, \lambda} g_{\boldsymbol{k} \lambda} \mathrm{e}^{\mathrm{i}\left(\omega_{\boldsymbol{k}}-E\right) t} b_{\boldsymbol{k}, \lambda}^{\dagger}+\text { h.c. }
$$

where $g_{\boldsymbol{k} \lambda}=\mathrm{i} \boldsymbol{d} \cdot \hat{e}_{\lambda}(\boldsymbol{k}) \sqrt{\omega_{\boldsymbol{k}} / 2 \epsilon_{0} \epsilon_{\mathrm{r}} v}, \boldsymbol{k}$ is a photon wave vector, $\omega_{\boldsymbol{k}}$ is the corresponding frequency, $\lambda$ denotes polarizations, $b_{\boldsymbol{k}, \lambda}, b_{\boldsymbol{k}, \lambda}^{\dagger}$ are photon annihilation and creation operators, $\boldsymbol{d}$ is the interband dipole moment (for simplicity equal for all $\mathrm{QDs}), \hat{e}_{\lambda}(\boldsymbol{k})$ is a unit polarization vector, $\epsilon_{0}$ is the vacuum permeability, $\epsilon_{\mathrm{r}}$ is the dielectric constant of the semiconductor, and $v$ is the normalization volume for the EM modes.

Finally, $H_{\mathrm{phot}}=\sum_{\boldsymbol{k}, \lambda} \hbar \omega_{\boldsymbol{k}} b_{\boldsymbol{k}, \lambda}^{\dagger} b_{\boldsymbol{k}, \lambda}$ is the Hamiltonian of the radiation reservoir.

To describe the ensemble of QDMs, we assume a Gaussian distribution $g\left(E_{1}, E_{2}\right)$ for the energies $E_{1}, E_{2}$ of the two dots, with mean values of the two energies $\bar{E}_{2}, \bar{E}_{2}$, identical energy variances $\sigma$ for both QDs, and a correlation coefficient 
$\rho$ between the energies $E_{1}$ and $E_{2}$ [5]. This corresponds to an uncorrelated Gaussian distribution of the parameters $E=\left(E_{1}+E_{2}\right) / 2$ and $\Delta=\left(E_{1}-E_{2}\right) / 2$ with variances $\sigma_{E}=\sigma \sqrt{(1+\rho) / 2}$ and $\sigma_{\Delta}=\sigma \sqrt{(1-\rho) / 2}$, respectively. In all our calculations we set $\sigma=8 \mathrm{meV}$. In the single-QDM model we fix $\Omega=\sqrt{V^{2}+\Delta^{2}}=11 \mathrm{meV}$ (that is, $\Delta$ is adjusted for each value of $V$ ). Similarly, in the QDM ensemble model, we fix $\bar{\Omega}=\sqrt{V^{2}+\bar{\Delta}^{2}}=11 \mathrm{meV}$.

We take the value of the spontaneous radiative decay rate for an individual QD to be $\Gamma=1 \mathrm{~ns}^{-1}$.

\section{The system evolution and optical response}

Optical experiments are modelled by exciting a QDM or an ensemble of QDMs with one or two ultrashort laser pulses, arriving at times $t_{i}$. In a linear experiment, there is only one pulse arriving at $t_{1}=0$. In the FWM experiments, two pulses are used, at times $t_{1}=-\tau$ and $t_{2}=0$. We assume that the pulses are spectrally very broad to assure resonance with all the QDs in the ensemble. If the durations of the pulses are much shorter than both $\hbar / \Delta$ and $\hbar / V$, the action of each of them corresponds to the instantaneous, independent rotation of the state of each QD, that is, to the unitary transformation $\mathrm{U}_{\mathrm{i}}=U_{i} \otimes U_{i}$, where

$$
U_{i}=\cos \left(\alpha_{i} / 2\right) I-\mathrm{i} \sin \left(\alpha_{i} / 2\right)\left[\mathrm{e}^{-\mathrm{i}\left(\phi_{i}+E t_{i}\right)}|0\rangle\langle 1|+\text { h.c. }\right]
$$

and $\alpha_{i}=\int_{-\infty}^{\infty} \mathrm{d} t f_{i}(t)$ is the pulse area.

Between the laser pulses, the evolution of the reduced density matrix of the charge subsystem is described by the Lindblad equation in the form [3]:

$$
\dot{\rho}=-\mathrm{i}\left[H_{\mathrm{X}}, \rho\right]+\mathcal{L}[\rho],
$$

with $\mathcal{L}[\rho]=\Gamma\left[\Sigma_{-} \rho \Sigma_{+}-(1 / 2)\left\{\Sigma_{+} \Sigma_{-}, \rho\right\}_{+}\right]$, where $\Sigma_{-}=\Sigma_{+}^{\dagger}=|0\rangle\langle 1|\otimes \mathbb{I}+\mathbb{I} \otimes| 0\rangle\langle 1|$. To find the state $\rho(t)$ for an arbitrary time $t$, we solve Eq. (5) as a usual vectorial differential equation in the Liouville space of $4 \times 4$ density matrices [6]. It turns out that the solution can be found analytically. Since $\Gamma \ll \bar{\Omega}$, we simplify the formulae by expanding the eigenvalues of the Liouville operator to the first and its eigenvectors to the second order in $\Gamma$.

The optical polarization of a single QDM under consideration is proportional to $P(t)=\langle 11|\rho(t)(|01\rangle+|10\rangle)+(\langle 01|+\langle 10|) \rho(t) \mid 00\rangle+$ c.c.

First, we study the coherent optical response from a single QDM upon excitation by a single pulse. The coherent radiation is proportional to the optical (interband) polarization $P_{\operatorname{lin}}(t)$ of the QDM taken to the linear order in the pulse area. The latter is

$$
P_{\operatorname{lin}}(t)=\mathrm{i} \frac{\alpha}{2} \mathrm{e}^{-\frac{\Gamma}{2} t}\left[-\left(\mathrm{e}^{H}+\mathrm{e}^{-H}\right)+\left(\mathrm{e}^{H}-\mathrm{e}^{-H}\right) \frac{V}{\Omega}\right],
$$

where $H=V \Gamma /(2 \Omega) t+\mathrm{i} \Omega t$.

The signal from a single QDM is dominated by optical beats on picosecond timescale due to the energy mismatch between the dots. In Fig. 1 we plot the value of $\left|P_{\text {lin }}(t)\right|^{2}$ averaged over these beats for three values of $V$. The inset shows the beats on a fine timescale. On long timescales (tens of picoseconds to 
nanoseconds) one observes an exponential decay for decoupled QDs (solid line) and an exponential decay with a twice larger rate for coupled identical dots (that is, for $\Delta=0$; dotted line). In the intermediate range of $V$, the decay is not exponential (dashed). A similar behavior was observed for the exciton occupation in a single QDM prepared in a superradiant state [3]. In both these cases, the decay is described by a combination of two contributions, one decaying with an increased (superradiant) rate $\Gamma(1+V / \Omega)$ and the other with a decreased (subradiant) rate $\Gamma(1-V / \Omega)$. This effect is a fingerprint of superradiant coupling of the two QDs forming the molecule to the radiation field.

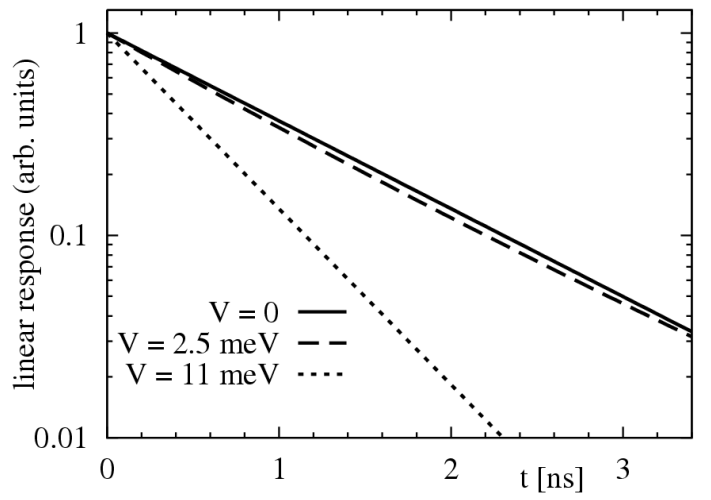

Fig. 1. Linear response as a function of time for the values of $V$ as shown.

The third order FWM response is given by $P_{\mathrm{FWM}}(t)=(\mathrm{i} / 4) \alpha_{1} \alpha_{2}^{2} \sum_{j} P_{j}(t)+$ c.c., where $P_{j}(t)$ are interband polarizations induced by two pulses, summed over the ensemble with the distribution function $g\left(E_{1}, E_{2}\right)$,

$$
\begin{aligned}
P_{1}(t) & =\frac{1}{2} \mathrm{e}^{-\frac{\Gamma}{2}(\tau+t)} \int \mathrm{d} E_{1} \mathrm{~d} E_{2} g\left(E_{1}, E_{2}\right) \mathrm{e}^{-\mathrm{i} E(t-\tau)}\left(\mathrm{e}^{H_{1}}+\mathrm{e}^{-H_{1}}\right) \\
\times & \frac{V^{2}+\Omega^{2}}{\Omega^{2}}, \\
P_{2}(t) & =\mathrm{e}^{-\frac{\Gamma}{2}(\tau+t)} \int \mathrm{d} E_{1} \mathrm{~d} E_{2} g\left(E_{1}, E_{2}\right) \mathrm{e}^{-\mathrm{i} E(t-\tau)}\left(-\mathrm{e}^{H_{1}}+\mathrm{e}^{-H_{1}}\right) \frac{V}{\Omega}, \\
P_{3}(t) & =-\frac{1}{2} \mathrm{e}^{-\frac{\Gamma}{2}(\tau+t)} \int \mathrm{d} E_{1} \mathrm{~d} E_{2} g\left(E_{1}, E_{2}\right) \mathrm{e}^{-\mathrm{i} E(t-\tau)} \\
\times & {\left[\left(\mathrm{e}^{H_{2}}+\mathrm{e}^{-H_{2}}\right) \frac{V^{2}}{\Omega^{2}}+\left(\mathrm{e}^{H_{2}}-\mathrm{e}^{-H_{2}}\right) \frac{V}{\Omega}\right], } \\
P_{4}(t) & =\mathrm{e}^{-\frac{\Gamma}{2}(\tau+3 t)} \int \mathrm{d} E_{1} \mathrm{~d} E_{2} g\left(E_{1}, E_{2}\right) \mathrm{e}^{-\mathrm{i} E(t-\tau)}\left(\mathrm{e}^{H_{3}}-\mathrm{e}^{-H_{3}}\right) \frac{V}{\Omega},
\end{aligned}
$$

where $\Omega=\sqrt{V^{2}+\Delta^{2}}$ and 


$$
\begin{aligned}
& H_{1}=\frac{V \Gamma}{2 \sqrt{\Delta^{2}+V^{2}}}(\tau+t)-\mathrm{i} \sqrt{\Delta^{2}+V^{2}}(\tau-t), \\
& H_{2}=\frac{V \Gamma}{2 \sqrt{\Delta^{2}+V^{2}}}(\tau-t)-\mathrm{i} \sqrt{\Delta^{2}+V^{2}}(\tau+t), \\
& H_{3}=\frac{V \Gamma}{2 \sqrt{\Delta^{2}+V^{2}}}(\tau+t)-\mathrm{i} \sqrt{\Delta^{2}+V^{2}}(\tau+t) .
\end{aligned}
$$

In our model, we include the heterodyne detection scheme with a reference pulse with the duration of $100 \mathrm{fs}$ full width at half maximum [5].

The FWM response, integrated over the real time $t$ is plotted in Fig. 2 as a function of the delay time between the pulses $\tau$. For $V \neq 0$ one observes beats at very short delay times (see the inset to Fig. 2a) which are due to optical interference of the coherent radiation emitted by the two QDs forming the QDM. These beats originate from the contribution from the terms $P_{3}(t)$ and $P_{4}(t)$, as discussed in our previous work [5]. These oscillations are damped on a timescale of $\tau \sim 1 / \sigma_{\Delta}$ because of the phase averaging due to inhomogeneous distribution of energy differences $\Delta$. They result in a considerable (a few percent) drop of the coherent polarization over a few picosecond time.

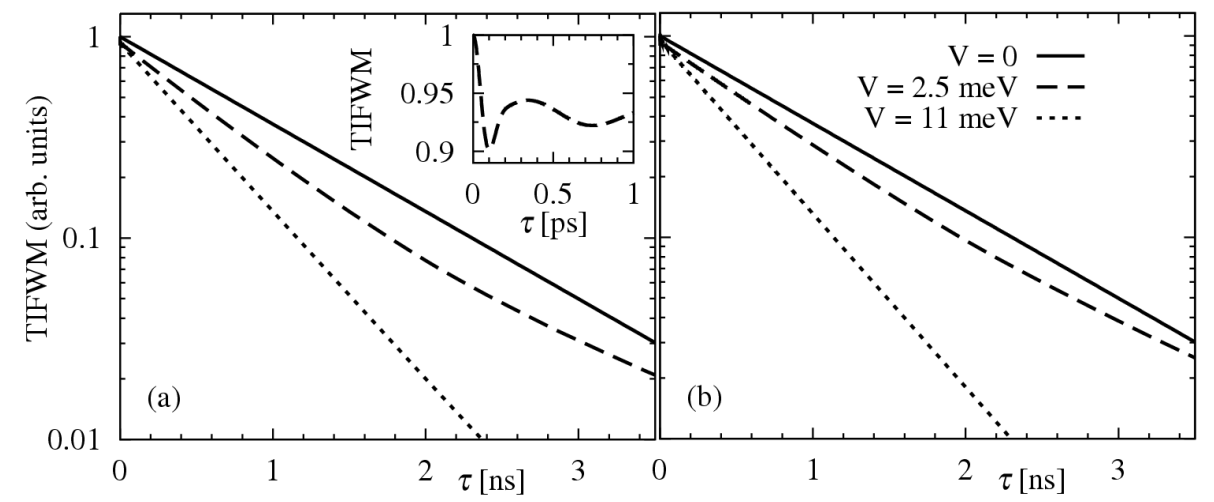

Fig. 2. Time-integrated FWM signal as a function of the delay for the values of $V$ as shown, for $\rho=0$ (a) and $\rho=0.875$ (b). The parameter values given in (b) are valid for both figures.

At longer delays, the time-integrated nonlinear response is dominated by radiative dephasing of the exciton states. Since the average energy mismatch $2 \bar{\Delta}$ is much larger than $\hbar \Gamma$, one cannot expect any collective effects for uncoupled dots $(V=0)$. This is confirmed by the results of our calculations (solid line in Fig. 2): indeed, the time-integrated FWM signal in this case decays exponentially with the rate $\Gamma$. In the opposite limit, $V=11 \mathrm{meV}, \bar{\Delta}=0$, the optical response decay is affected by the initial coherence drop, as discussed above, and the decay rate increases to $2 \Gamma$ (dotted line in Fig. 2). This shows that the collective (superradiant) coupling of the two dots in a QDM to the radiation field survives in spite of the inhomogeneity of the energy differences $\Delta$. 
The transition between these two regimes is driven by the interplay of the energy mismatch between the QDs forming the molecule and the coupling between them. In the intermediate range, the decay rate is roughly intermediate between these two limiting values but the process is in fact non-exponential. Moreover, it cannot be approximated by a sum of two exponentially decaying contributions, unlike in the case of the linear response from a single QDM. The evolution for these intermediate ratios of $V / \Omega$ depends on the statistical distribution of the QDM parameters. By comparing Figs. 2a and b one can see that the decay becomes faster for weakly correlated energies, that is, the smaller $\rho$, hence the larger $\sigma_{\Delta}$.

\section{Conclusions}

We have analyzed the linear and time-integrated FWM response from a single QD and an ensemble of QDs, respectively. Two features can be observed. One is an increase in the decay rate when the coupling strength dominates over the transition energy mismatch, which is a signature of collective (superradiant) emission of radiation. We observe a transition from the usual decay rate (uncoupled, different dots) to the superradiant, double rate (strongly coupled dots).

The other effect is a strong oscillation at very short (picosecond) delay times which nearly instantaneously reduces the amplitude of the FWM response by a few per cent whenever the dots are coupled. This effect results from damping of the optical coherence between the QDs due to a spread of energy mismatch across the ensemble. In a real system, this signal drop will add to the phonon-induced pure dephasing effect, which may explain the increased initial dephasing observed in the experiment [1].

Although qualitatively the linear and nonlinear responses are similar, there is no strict relation between them. In particular, the optical beats in the single-QDM signal are replaced by strongly damped oscillations and coherence drop in the ensemble case. Moreover, the decay of coherence for intermediate couplings is determined by the coupling strength in the single-QD case, while it strongly depends on the ensemble distribution of the energy mismatch in the FWM case.

\section{References}

[1] B.D. Gerardot, S. Strauf, M.J.A. de Dood, A.M. Bychkov, A. Badolato, K. Hennessy, E.L. Hu, D. Bouwmeester, P.M. Petroff, Phys. Rev. Lett. 95, 137403 (2005).

[2] E. Rozbicki, P. Machnikowski, Phys. Rev. Lett. 100, 027401 (2008).

[3] A. Sitek, P. Machnikowski, Phys. Rev. B 75, 035328 (2007).

[4] P. Borri, W. Langbein, U. Woggon, M. Schwab, M. Bayer, S. Fafard, Z. Wasilewski, P. Hawrylak, Phys. Rev. Lett. 91, 267401 (2003).

[5] A. Sitek, P. Machnikowski, Acta Phys. Pol. A 112, 167 (2007).

[6] S. Mukamel, Principles of Nonlinear Optical Spectroscopy, Oxford University Press, Oxford 1995. 